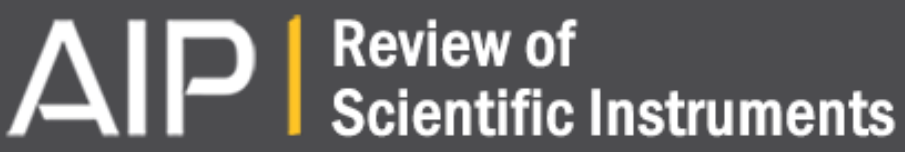

The evaluation of phasemeter prototype performance for the space gravitational waves detection

He-Shan Liu, Yu-Hui Dong, Yu-Qiong Li, Zi-Ren Luo, and Gang Jin

Citation: Review of Scientific Instruments 85, 024503 (2014); doi: 10.1063/1.4865121

View online: http://dx.doi.org/10.1063/1.4865121

View Table of Contents: http://scitation.aip.org/content/aip/journal/rsi/85/2?ver=pdfcov

Published by the AIP Publishing

\section{Articles you may be interested in}

Toward gravitational wave detection

AIP Conf. Proc. 523, 451 (2000); 10.1063/1.1291910

Gravitational wave detection-the way forward

AIP Conf. Proc. 523, 376 (2000); 10.1063/1.1291882

Detection of gravitational waves from eccentric compact binaries

AIP Conf. Proc. 493, 48 (1999); 10.1063/1.1301562

What will we learn from the detection of gravitational waves?

AIP Conf. Proc. 493, 25 (1999); 10.1063/1.1301560

Confusion noise estimate for gravitational wave measurements in space

AIP Conf. Proc. 456, 68 (1998); 10.1063/1.57424

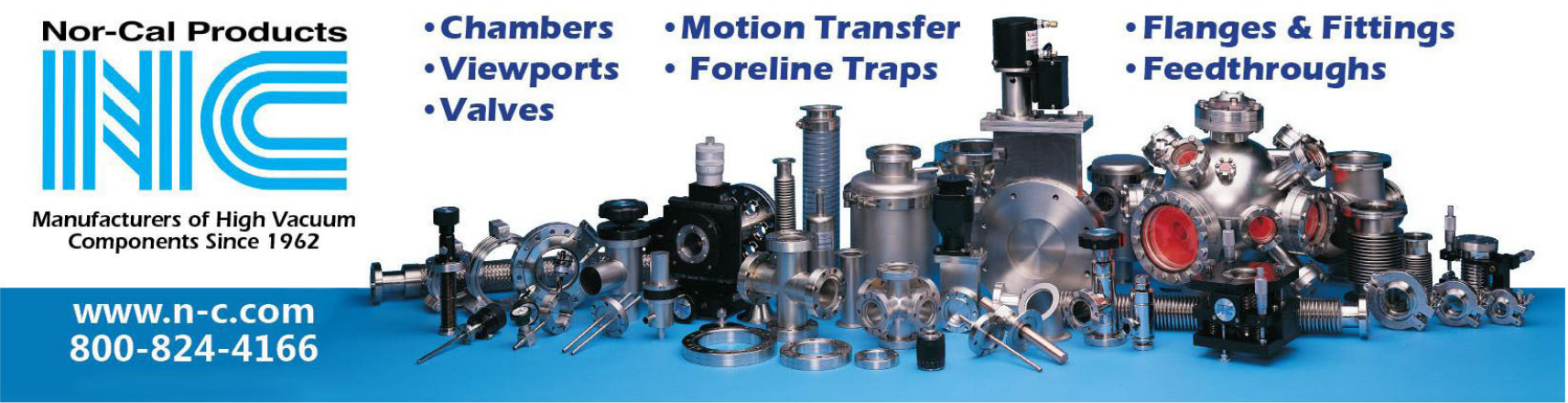




\title{
The evaluation of phasemeter prototype performance for the space gravitational waves detection
}

\author{
He-Shan Liu, ${ }^{1,2, a)}$ Yu-Hui Dong, ${ }^{1,2, a)}$ Yu-Qiong Li, ${ }^{1}$ Zi-Ren Luo, ${ }^{1}$ and Gang $\mathrm{Jin}^{1, \mathrm{~b})}$ \\ ${ }^{1}$ National Microgravity Laboratory (NML), Institute of Mechanics, Chinese Academy of Sciences, Beijing \\ 100190, People's Republic of China \\ ${ }^{2}$ University of Chinese Academy of Sciences, Beijing 100190, People's Republic of China
}

(Received 5 December 2013; accepted 26 January 2014; published online 14 February 2014)

\begin{abstract}
Heterodyne laser interferometry is considered as the most promising readout scheme for future space gravitational wave detection missions, in which the gravitational wave signals disguise as small phase variances within the heterodyne beat note. This makes the phasemeter, which extracts the phase information from the beat note, the key device to this system. In this paper, a prototype of phasemeter based on digital phase-locked loop technology is developed, and the major noise sources which may contribute to the noise spectra density are analyzed in detail. Two experiments are also carried out to evaluate the performance of the phasemeter prototype. The results show that the sensitivity is achieved $2 \pi \mu \mathrm{rad} / \sqrt{ } \mathrm{Hz}$ in the frequency range of $0.04 \mathrm{~Hz}-10 \mathrm{~Hz}$. Due to the effect of thermal drift, the noise obviously increases with the frequencies down to $0.1 \mathrm{mHz}$. ( $) 2014$ AIP Publishing LLC. [http://dx.doi.org/10.1063/1.4865121]
\end{abstract}

\section{INTRODUCTION}

In the quest of searching gravitational waves, the evolved Laser Interferometer Space Antenna (eLISA) is designed to look for the signals emitting from the super-mass black holes in-spirals harbored in oldest galaxies or the black hole binaries formed by the relic of pop III stars. ${ }^{1,2}$ The information of the gravitational waves will be disguised as phase changes embed in the eLISA's interferometric beat notes. ${ }^{3-5}$ Due to the orbital drifting, the frequencies of the beat notes will range from $5 \mathrm{MHz}$ to $25 \mathrm{MHz}{ }^{6}$ Within the eLISA's most sensitive frequency band from $0.1 \mathrm{mHz}$ to $1 \mathrm{~Hz}$, the phasemeter used for the eLISA mission is required to be able to extract the phase from input signal with additional phase noise no greater than $2 \pi \mu \mathrm{rad} / \sqrt{ } \mathrm{Hz} .{ }^{6,7}$ Various types of phase measurement techniques have been considered for eLISA mission. In the early years, the phasemeter using zero-crossing technology has been demonstrated, but only for single fixed frequency input. ${ }^{8-11}$ While the most suitable phasemeter architecture, which can provide the required accuracy, is based on a socalled digital phase-locked loop (DPLL) ${ }^{12-14}$ Inspired by the eLISA and related missions (LISA, ${ }^{15,16}$ LISA pathfinder, ${ }^{17,18}$ etc.), some phasemeter prototypes of phase readout precision up to $2 \pi \mu \mathrm{rad} / \sqrt{ } \mathrm{Hz}$ in the frequency band from $1 \mathrm{mHz}$ to $10 \mathrm{~Hz}$ have been reported in Albert Einstein Institute ${ }^{12,13}$ (AEI, Germany) and Jet Propulsion Laboratory ${ }^{14}$ (JPL, USA). Recently, an elegant breadboard model of the LISA phasemeter is currently under development, and the performance goals for the phasemeter is to achieve $2 \pi \mu \mathrm{rad} / \sqrt{ } \mathrm{Hz}$ in the range of $0.1 \mathrm{mHz}-1 \mathrm{~Hz}{ }^{19,20}$

With the developing interests of China in the field of space gravitational wave detection, a ground demonstration of laser interferometer has been built. ${ }^{21}$ Try to understand the

\footnotetext{
a) H.-S. Liu and Y.-H. Dong contributed equally to this work.

b) Author to whom correspondence should be addressed. Electronic mail: gajin@imech.ac.cn.
}

problems that limit the performance of phasemeter and build the phasemeter for Chinese space gravitational wave detection mission, ${ }^{22}$ a prototype of phasemeter has been constructed in the paper. The architecture description and the noise sources analysis of the phasemeter are also included. In addition, two experiments that the phasemeter test on internal and external signals will be presented to evaluate the phasemeter's performance.

\section{DESIGN AND ARCHITECTURE}

The architecture of the phasemeter prototype shown in Fig. 1 was implemented on a commercial Field Programmable Gate Array (FPGA) platform (Terasic, DE3-340). The detected signals are digitized by analog to digital converters (ADCs) (AD9254) and then multiplied by a numerically controlled oscillator (NCO) with quadrature signals. After that, the mixed signals are filtered by the low-pass filter (LPF) to remove the multiplied frequency components. The cutoff and sampling frequencies of the LPF are, respectively, $1 \mathrm{MHz}$ and $40 \mathrm{MHz}$. Limited by the LPF, the frequencies of the tested signal should be restricted in the range of $500 \mathrm{KHz}-10 \mathrm{MHz}$. The intensity of the output signal after filtering is proportional to the phase difference between the detected signal and the $\mathrm{NCO}$, and the phase will be reconstructed by arctangent calculation parts. The phase changes resulted from the frequency error between the detected signal and $\mathrm{NCO}$ are used to update the NCO frequency to keep the DPLL frequency locked to the incoming signal. Finally, the two phase signals are subtracted to remove the common-mode noise, thus the phase difference information can be obtained. The FPGA board is driven by an ultra-stable oscillator (USO) (Xi' an Hongtai time-frequency, China), of which the stability and accuracy are, respectively, $10^{-12}$ and $5 \times 10^{-11}$ in the time range of $1-10000 \mathrm{~s}$. Fig. 2 shows the physical picture of the phasemeter prototype. 


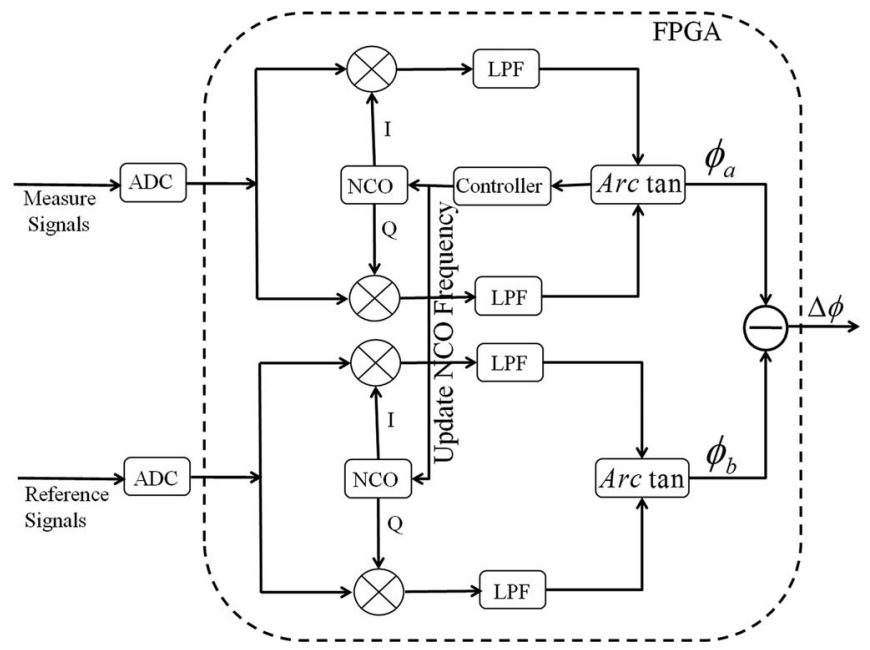

FIG. 1. Schematic diagram of the phasemeter prototype.

\section{NOISES ANALYSIS}

Assuming an input signal $S_{0}$ with amplitude $A$, frequency $\omega$, and initial phase $\varphi_{0}$, the detected signal can be written as

$$
S_{0}=A \sin \left(\omega t+\varphi_{0}\right) \text {. }
$$

If considering all the noises, such as the laser frequency jitter noise $\delta \Phi$ and electronic noise, the expression (1) can be modified to

$$
S_{0}=A \sin \left(\omega t+\delta \Phi+\varphi_{0}\right)+\delta_{A},
$$

where $\delta_{A}$ expresses the sum of the electronic noise, and other noises which present the property of white noise. Before access to the FPGA, the detected signals need to pass through the analog frontend and be quantized by the ADCs. The tested signals will have phase shifts when pass through the analog frontend, and the phase shifts also vary along with the temperature and frequency changing. Besides, the ADCs will import the quantization noise $\sigma_{q}$, sampling jitter noise $\delta_{\varphi}$, and the electronic noise. So, the expression (2) is modified as

$$
S_{0}=A \sin \left(\omega t+\delta \Phi+\varphi_{0}+\delta_{\varphi}+\sigma_{q}+\varphi(T, \omega)\right)+\delta_{A}{ }^{*} .
$$

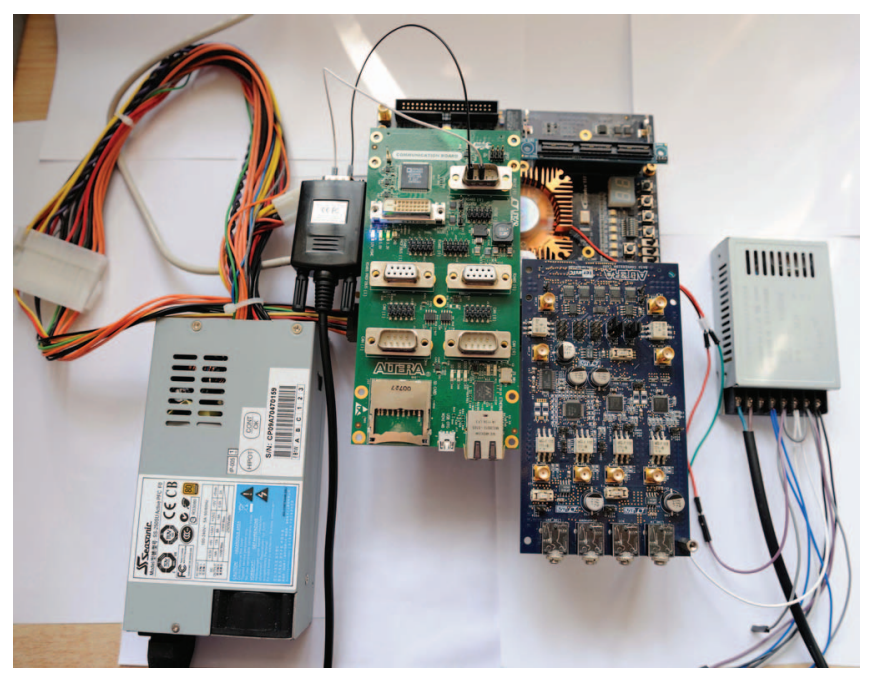

FIG. 2. Physical picture of the phasemeter prototype.
$\delta_{A}{ }^{*}$ is the modification of $\delta_{A}$ which adds the electronic noise caused by the ADC. With the $R$ as the sign of NCO signals and also considering the frequency error $\Delta \omega t$ imported by the feedback loop, the signals of NCO can be expressed by

$$
\begin{gathered}
R_{\mathrm{cos}}=\cos \left(\omega t+\Delta \omega t+\varphi_{1}\right), \\
R_{\sin }=\sin \left(\omega t+\Delta \omega t+\varphi_{1}\right) .
\end{gathered}
$$

The detected signals $S_{0}$ will be, respectively, multiplied by the NCO signals, which two mixed signals can be obtained in the following:

$$
\begin{aligned}
S_{0} \times R_{\cos }= & \left(A \sin \left(\omega t+\delta \Phi+\varphi_{0}+\delta_{\varphi}+\sigma_{q}+\varphi(T, \omega)\right)+\delta_{A}{ }^{*}\right) \\
& \times \cos \left(\omega t+\Delta \omega t+\varphi_{1}\right) \\
= & A / 2\left(\operatorname { s i n } \left(\omega t+\delta \Phi+\varphi_{0}+\delta_{\varphi}+\sigma_{q}+\varphi(T, \omega)\right.\right. \\
& \left.+\omega t+\Delta \omega t+\varphi_{1}\right)+\sin \left(\omega t+\delta \Phi+\varphi_{0}+\delta_{\varphi}+\sigma_{q}\right. \\
& \left.\left.+\varphi(T, \omega)-\omega t-\Delta \omega t-\varphi_{1}\right)\right)+\delta_{A}{ }^{*} \\
& \times \cos \left(\omega t+\Delta \omega t+\varphi_{1}\right), \\
S_{0} \times R_{\sin }= & \left(A \sin \left(\omega t+\delta \Phi+\varphi_{0}+\delta_{\varphi}+\sigma_{q}+\varphi(T, \omega)\right)+\delta_{A}{ }^{*}\right) \\
& \times \sin \left(\omega t+\Delta \omega t+\varphi_{1}\right) \\
= & A / 2\left(-\cos \left(\omega t+\delta \Phi+\varphi_{0}+\delta_{\varphi}+\sigma_{q}+\varphi(T, \omega)\right.\right. \\
& \left.+\omega t+\Delta \omega t+\varphi_{1}\right)+\cos \left(\omega t+\delta \Phi+\varphi_{0}+\delta_{\varphi}+\sigma_{q}\right. \\
& \left.\left.+\varphi(T, \omega)-\omega t-\Delta \omega t-\varphi_{1}\right)\right)+\delta_{A}{ }^{*} \\
& \times \sin \left(\omega t+\Delta \omega t+\varphi_{1}\right) .
\end{aligned}
$$

Then, suppressing by appropriate LPF, the expressions (5a) and $(5 b)$ are simplified to

$P=A / 2 \sin \left(\delta \Phi-\Delta \omega t+\varphi_{0}+\delta_{\varphi}+\sigma_{q}-\varphi_{1}+\varphi(T, \omega)\right)+\sigma_{A}$,

$Q=A / 2 \cos \left(\delta \Phi-\Delta \omega t+\varphi_{0}+\delta_{\varphi}+\sigma_{q}-\varphi_{1}+\varphi(T, \omega)\right)+\sigma_{A}$,

where $\sigma_{A}$ is the value of the multiplication part and the last noise part after filtering.

Finally, the phase $\phi$ will be obtained by the arctangent part,

$$
\phi=\left(\varphi_{0}-\varphi_{1}\right)+\delta \Phi-\Delta \omega t+\delta_{\varphi}+\varphi(T, \omega)+\sigma_{q}+\sigma_{\varphi} .
$$

The phase $\phi$ includes the phase information $\varphi_{0}-\varphi_{1}$, laser frequency jitter noise of the tested signal $\delta \Phi$, frequency error $\Delta \omega t$ between the tested signal and the NCO caused by the feedback loop, the sampling jitter noise of $\operatorname{ADC} \delta_{\varphi}$, phase shift of the analog frontend $\varphi(T, \omega)$, quantization noise $\sigma_{q}$, and the remaining noises $\sigma_{\varphi}$. In the following, the above noise will be, respectively, discussed.

\section{A. Phase information}

The phase information is the message which contains the gravitational waves. However, it is difficult to distinguish phase information from noise, especially the noise come from the environment which has the similar frequencies and amplitude, such as the vibration noise and thermal noise. In order to 
quantify the noise level of phasemeter itself, we split one signal into two halves and feed into two channels, the difference of the two outputs are used to represent the intrinsic noise of phasemeter.

\section{B. Frequency noise}

In the interferometer, frequency noise $\delta \Phi$ is caused by the frequency jitter of laser. Due to the unequal arm-length of interferometer, the frequency noise will be largely increased and becomes primary noise in the eLISA's interferometric beat notes. Converting into displacement variance $\delta l=\frac{\delta \Phi}{2 \pi} \lambda$, frequency fluctuation noise can be calculated by the equation ${ }^{5}$

$$
\delta l=\Delta L \frac{\delta \mu}{\mu} .
$$

$\Delta L$, the length difference between interferometer arm; $\delta \mu$, the value of frequency instability; $\mu$, the frequency of laser; $\lambda$, the wavelength of laser.

Differently, the instability of driving clock determines the value of frequency noise in the paper, where the detected signal is produced by a function generator. In this situation, the frequency noise is similar with the sampling jitter noise of ADC.

\section{Sampling jitter noise}

The ADC is driven by local oscillator. When sampling the signal with ADC, the frequency variance of local oscillator will introduce phase noise into sampled signal. This noise is called sampling jitter noise ${ }^{14}$

$$
\delta_{\varphi}=\delta_{t} \times f_{b} .
$$

$\delta_{t}$, the sampling time error of $\mathrm{ADC} ; f_{b}$, the frequency of tested signal. In our phasemeter, the stability of the USO is better than $10^{-12}$ in the time range of $1-10000 \mathrm{~s}$. For a $1 \mathrm{MHz}$ signal, the absolute value of time jitter noise is no more than $2 \pi \times 10^{-6} \mathrm{rad}$.

\section{Quantization noise}

The quantization noise $\sigma_{q}$ can be calculated in Eq. (10), and the noise is a function of sampling rate $f_{s}$ and number of bits $N .{ }^{14}$ With a 14 bit ADC sampling at $100 \mathrm{MHz}$ in our phasemeter prototype, the quantization noise is less than $10^{-8} \mathrm{rad} / \sqrt{ } \mathrm{Hz}$ :

$$
\sigma_{q}=\frac{1}{\pi 2^{N} \sqrt{6 f_{s}}} .
$$

\section{E. Feedback loop noise}

Feedback loop noise is produced by the NCO being unable to perfectly track the frequency of tested signal. Due to the orbital drifting, the frequencies of the beat notes will range from $5 \mathrm{MHz}$ to $25 \mathrm{MHz}{ }^{6}$ That huge frequency fluctuation and low signal to noise ratio (SNR) of interferometric signal in the space mission will be rigorous challenges to the feedback loop. Optimizing the feedback loop algorithm is the only way to decrease the noise, and also an important research area in the phasemeter for the future. If we neglect the instability of driving clock in the paper, the frequency of the detected signal is a constant. So, the feedback loop noise will be small enough, and the experiment results in Sec. IV also verify the analysis.

\section{F. Analog frontend noise}

Signals will have phase shifts when pass through the analog circuit, and the phase shifts also vary along with the frequency changing and the temperature drift. The analog circuits of different channels of phasemeter have minor differences, such as length, spurious capacitance, and inductance, which are sensitive to the temperature and frequency changing. Due to the Doppler effect of between two satellites, the frequency of interferometer signal varies greatly. Moreover, the satellites also face the high amplitude of temperature fluctuation. So, some methods should be carried out to remove the noise which likely submerges to the real information.

\section{G. Other noises}

Other noises mainly come from the electronic noise which may be characterized as white noise in the gravitational wave frequency ranges. The electronic parts and components, especially the analog device, can introduce electronic noise. Therefore, the components selection and the circuits design are also a significant work in phasemeter building. The noise also includes the calculation error in FPGA which is due to its limited hardware resources.

In the process of noise analysis, we will not go deep into the optical path noise and the acceleration noise. The optical path noise includes shot noise and beam pointing noise. The acceleration noise is raised from proof mass's disturbance. Both of them directly enter the final phase readout. However, in the paper we only focus on the intrinsic noise of phasemeter. The tested signals we used are produced by a functional generator, which do not contain optical path noise and acceleration noise.

\section{EXPERIMENT RESULTS AND DISCUSSIONS}

\section{A. Internal signal test}

Fig. 3 shows the results of first experiment, where the detected signal was generated by an additional NCO implemented in the same FPGA of the phasemeter. The purpose was to verify the functionality and noise level of the phasemeter prototype in ideal situation.

From Eq. (7), the phase $\phi_{a}$ and $\phi_{b}$ can be expressed, respectively, by

$$
\begin{aligned}
& \phi_{a}=-\Delta \omega t+\sigma_{\varphi a} \\
& \phi_{b}=-\Delta \omega(t+\delta t)+\sigma_{\varphi b} .
\end{aligned}
$$

So, the phase difference is

$$
\phi_{a}-\phi_{b}=-\Delta \omega \times \delta t+\sigma_{\varphi} .
$$




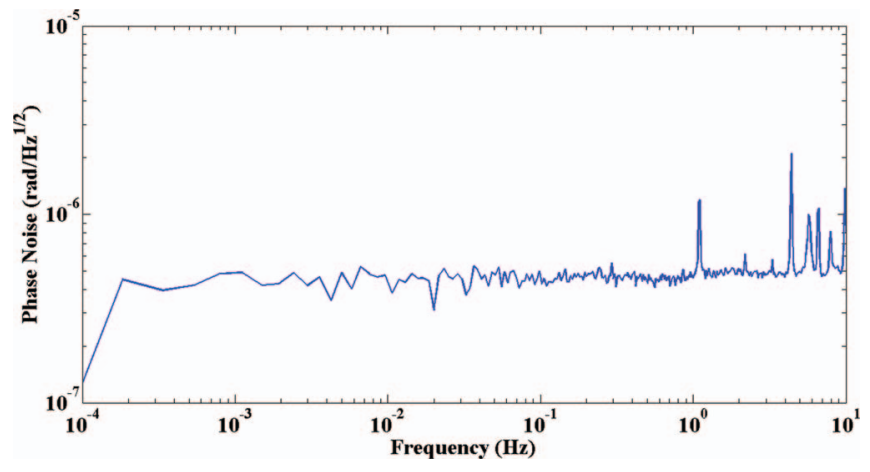

FIG. 3. Noise performance of the phasemeter with $1 \mathrm{MHz}$ input signals were generated by an additional NCO implemented in FPGA. And, the initial frequency of the phasemeter $\mathrm{NCO}$ was $1 \mathrm{MHz}$ minus $0.5 \mathrm{~Hz}$. The results had been smoothed by the method of linear amplitude spectrum density (LASD).

$\delta t$ represents the time shifts difference between two channels of the phasemeter prototype, $\Delta \omega$ will be compensated by the feedback loop, where

$$
\sigma_{\varphi}=\sqrt{\sigma_{\varphi a}^{2}+\sigma_{\varphi b}^{2}} .
$$

The expression (12) indicates that the phase error will be proportional to the frequency errors variation, and the phase error can be suppressed if the feedback loop works more precisely. Seeing from Fig. 3, the noise curve except for some peaks is flat over the frequency range from $0.1 \mathrm{mHz}$ to $10 \mathrm{~Hz}$, and the noise level is only limited by the computing error of the FPGA. The peaks arise at $1 \mathrm{~Hz}$ and its multiples come from the residue of frequency compensation of feedback loop.

\section{B. External signal test}

In the second experiment, the detected signals with different frequencies were generated by an external function generator (Agilent, 33522A). Besides, the function generator had been synchronized by the same reference clock with the FPGA board to remove the relative frequency and phase error between the detected signals and the NCO. Before accessed to the FPGA, the external signal needed to pass through analog frontend and be digitized by ADCs, so that all the hardware could be tested. In this experiment, the frequency range was chosen from $500 \mathrm{KHz}$ to $3 \mathrm{MHz}$. The experiment results were shown in Fig. 4.

In this experiment, all the noise sources described in expression (7) are all included. So, the phase difference is

$$
\begin{aligned}
\phi_{a}-\phi_{b}= & \delta \Phi-\Delta \omega \times \delta t+\delta_{\varphi}+\left(\varphi_{a}(T, \omega)\right. \\
& \left.-\varphi_{b}(T, \omega)\right)+\sigma_{q}+\sigma_{\varphi} .
\end{aligned}
$$

The fitting line in Fig. 4(a) shows that the phase noise $\varphi_{a}(\omega)$ $-\varphi_{b}(\omega)$ increases along with the frequency of input signal linearly. This result may apply to any analog circuits as well as the analog frontend of the phasemeter. From Fig. 4(b) and expression (14), the noise floor of Fig. 3(b) is naturally submerged by the greater noise. But it is also shown that the phase sensitivity is achieved $2 \pi \mu \mathrm{rad} / \sqrt{ } \mathrm{Hz}$ in higher frequency range $(0.04-10 \mathrm{~Hz})$. While the phase noise increase
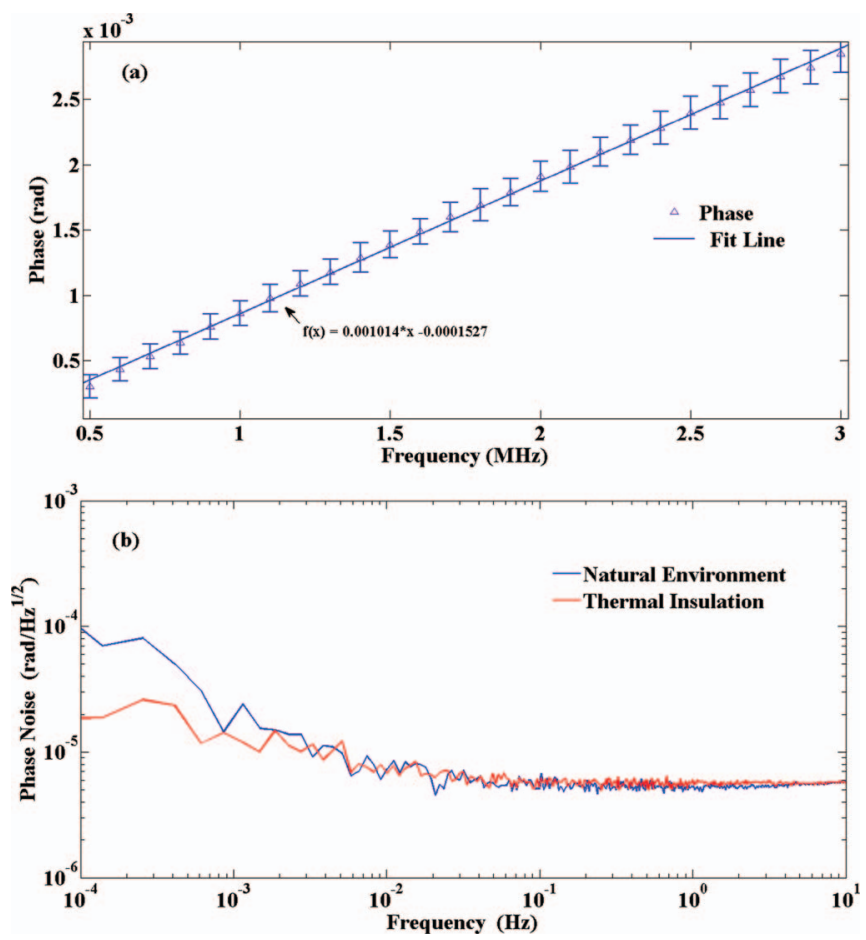

FIG. 4. Noise performance of the phasemeter with various frequencies input signals generated by an external function generator: (a) phase information with different frequencies; (b) typical noise performs with $1 \mathrm{MHz}$ signal input, and the data had also been smoothed by the method of LASD. The natural environment was the conditions without any thermal insulation methods in our laboratory, where the temperature fluctuated about a few degrees. The temperature variation of the thermal insulation conditions which used some simple thermal insulation methods was no more than $0.2^{\circ} \mathrm{C}$ (tested by Fluke 54-IIB).

at the lower frequency range $(0.04 \mathrm{~Hz}-0.1 \mathrm{mHz})$ which will be discussed below.

Quantization noise $\sigma_{q}$ has been calculated in Sec. III, and the feedback loop noise $\Delta \omega \times \delta t$ is small enough which has been tested in Fig. 3. The noise $\sigma_{\varphi}$ mainly includes the electronic noise which can be characterized as white noise; it may have the same amplitude $2 \pi \mu \mathrm{rad}$ at low frequencies as it has at high frequencies. According to the expression (14), the remaining reasons which result in the low frequency phase noises enlarge will be analyzed in the following: (1) One of the noises possibly come from the fluctuation of signal frequency produced by the functional generator $\varphi_{a}(\omega)$ $-\varphi_{b}(\omega)$. But the function generator has been synchronized by the same reference clock with the FPGA board, and the stability of USO is $10^{-12}$ in the time range of $1-10000 \mathrm{~s}$. It means that the fluctuation degree of tested signal is lower than $10^{-5} \mathrm{~Hz}$. According to the results of Fig. 4(a), the phase noise $\varphi_{a}(\omega)-\varphi_{b}(\omega)$ caused by $10^{-5} \mathrm{~Hz}$ fluctuation is no more than $10^{-14} \mathrm{rad}$ in the experiment. Therefore, the noise source is not the key element of the low frequency noise. (2) The value of phase shift $\varphi_{a}(T)-\varphi_{b}(T)$ will be varied along with the environment especially the temperature fluctuation. According to the results in Fig. 4(b), reducing the temperature fluctuation can obviously decrease the noise level, especially in the frequency range of $1-0.1 \mathrm{mHz}$. So, the thermal drift is the main contribution to the lower frequency phase noise. This will be quantitatively analyzed in our future work. (3) The sampling 
jitter noise of $\operatorname{ADC} \delta_{\varphi}$ and the frequency jitter noise $\delta \Phi$ of tested signal possibly are one of the reasons. Unfortunately, the frequency jitter performance of the USO in power spectral density (PSD) has not been provided. However, according to the value calculated in Sec. III C, it could confirm that the reason is not the primary one.

From the above analysis, the phase noise in lower frequency is mainly caused by the thermal drift of environment. Reducing the temperature fluctuation and decreasing the imbalance of analog frontend are the main methods to eliminate the noise, but need us to make further research.

\section{CONCLUSIONS}

The phase noise of the phasemeter based on the DPLL architecture has been analyzed in detail in the paper. Moreover, a phasemeter prototype has been developed and tested with the internal and external signal resources to demonstrate the functionality and the noise performance. Under the situation of external testing signals, the phase sensitivity is achieved $2 \pi \mu \mathrm{rad} / \sqrt{ } \mathrm{Hz}$ in the frequencies from $0.04 \mathrm{~Hz}$ to $10 \mathrm{~Hz}$, and the noise increase obviously in the frequency below $0.04 \mathrm{~Hz}$. How to reduce the low frequency phase noise mainly caused by the thermal drift is currently the main subject of investigations.

The prototype developed in this paper cannot meet the requirement of the eLISA mission to extract the length information, because the eLISA cannot introduce the reference optical path. However, the prototype can be used for beam pointing mission ( $\geq$ four channels) and measuring the relative moving between the testing mass and optical bench in the ground interferometer demonstration. The prototype will be demonstrated in heterodyne laser interferometer for further analysis, and the process of identifying and removing noise to make the phasemeter meet the eLISA or related missions requirements in China will be continued.

\section{ACKNOWLEDGMENTS}

This work was financially supported by the Scientific Equipment Development and Research Project of Chinese Academy of Sciences (Project Code: Y231411YB1), and also supported by the Space Science Research Projects in
Advance of Chinese Academy of Sciences (Project Code: O930143XM1).

${ }^{1}$ P. Amaro-Seoane, S. Aoudia, S. Babak, P. Binétruy, E. Berti, A. Bohé, C. Caprini, M. Colpi, N. J. Cornish, K. Danzmann et al., Class. Quantum Grav. 29(12), 124016 (2012).

${ }^{2}$ P. Binétruy, A. Bohé, C. Caprini, and J. F. Dufaux, J. Cosmol. Astropart. Phys. 06, 027 (2012).

${ }^{3}$ P. R. Saulson, Fundamentals of Interferometric Gravitational Wave Detectors (World Scientific, Singapore, 1994), pp. 12-18.

${ }^{4}$ M. Pitkin, S. Reid, S. Rowan, and J. Hough, Living Rev. Relativ. 14(5), 13-20 (2011).

${ }^{5}$ K. Danzmann, P. Bender, A. Brillet, I. Ciufolini, A. M. Cruise, C. Cutler, F. Fidecaro, W. M. Folkner, J. Hough, P. McNamara et al., "LISA Pre-Phase A Report," 2nd ed., Max-Planck-Institut fur Quantenoptik Report No. MPQ 208, Garching, Germany, 1998, pp. 57-61.

${ }^{6}$ P. Amaro-Seoane, G. Auger, S. Babak, M. Bassan, K. Belczynski, P. Binetruy, J. Bogenstahl, D. Bortoluzzi, C. Caprini, A. Cavalleri et al., eLISAWhitepaper-RC1 (eLISA Working Group, 2013), pp. 13-14.

${ }^{7}$ O. Jennrich, P. Binetruy, M. Colpi, K. Danzmann, P. Jetzer, A. Lobo, G. Nelemans, B. Schutz, R. Stebbins, T. Sumner et al., The eLISA/NGO Whitepaper (eLISA Working Group, 2011), pp. 88-89.

${ }^{8}$ O. Jennrich, R. T. Stebbins, P. L. Bender, and S. Pollack, Class. Quantum Grav. 18, 4159-4164 (2001)

${ }^{9}$ S. E. Pollack, O. Jennrich, R. T. Stebbins, and P. Bender, Class. Quantum Grav. 20, S193-S199 (2003).

${ }^{10}$ S. E. Pollack and R. T. Stebbins, Class. Quantum Grav. 23, 4189-4200 (2006).

${ }^{11}$ V. Wand, F. Guzmán, G. Heinzel, and K. Danzmann, AIP Conf. Proc. 873, 689 (2006).

${ }^{12}$ I. Bykov, J. J. E. Delgado, A. F. G. Marín, G. Heinzel, and K. Danzmann, J. Phys.: Conf. Ser. 154, 012017 (2009).

${ }^{13}$ G. Heinzel, V. Wand, A. García, O. Jennrich, C. Braxmaier, D. Robertson, K. Middleton, D. Hoyland, A. Rüdiger, R. Schilling et al., Class. Quantum Grav. 21, S581-S587 (2004).

${ }^{14}$ D. Shaddock, B. Ware, P. G. Halverson, and B. Klipstein, AIP Conf. Proc. 873, 654-660 (2006)

${ }^{15}$ F. G. Cervantes, R. Flatscher, D. Gerardi, J. Burkhardt, R. Gerndt, M. Nofrarias, J. Reiche, G. Heinzel, K. Danzmann, L. G. Boté et al., Astron. Soc. Pacific Conf. Ser. 467, 141 (2013).

${ }^{16}$ K. Danzmann and A. Rüdiger, Class. Quantum Grav. 20(10), S1 (2003).

${ }^{17}$ F. Antonucci, M. Armano, H. Audley, G. Auger, M. Benedetti, P. Binetruy, C. Boatella, J. Bogenstahl, D. Bortoluzzi, P. Bosetti et al., Class. Quantum Grav. 28(9), 094001 (2011).

${ }^{18}$ P. McNamara, S. Vitale, and K. Danzmann, Class. Quantum Grav. 25(11), 114034 (2008).

${ }^{19}$ O. Gerberding, S. Barke, I. Bykov, K. Danzmann, A. Enggaard, J. J. Esteban, A. Gianolio, T. V. Hansen, G. Heinzel, A. Hornstrup et al., preprint arXiv:1208.6418 (2012)

${ }^{20}$ O. Gerberding, B. Sheard, I. Bykov, J. Kullmann, J. J. E. Delgado, K. Danzmann, and G. Heinzel, Class. Quantum Grav. 30, 235029 (2013).

${ }^{21}$ Y.-Q. Li, Z.-R. Luo, H.-S. Liu, Y.-H. Dong, and G. Jin, Chin. Phys. Lett. 29, 079501 (2012)

${ }^{22}$ X. Gong, S. Xu, S. Bai, Z. Cao, G. Chen, Y. Chen, X. He, G. Heinzel, Y. Lau, C. Liu et al., Class. Quantum Grav. 28(9), 094012 (2011). 